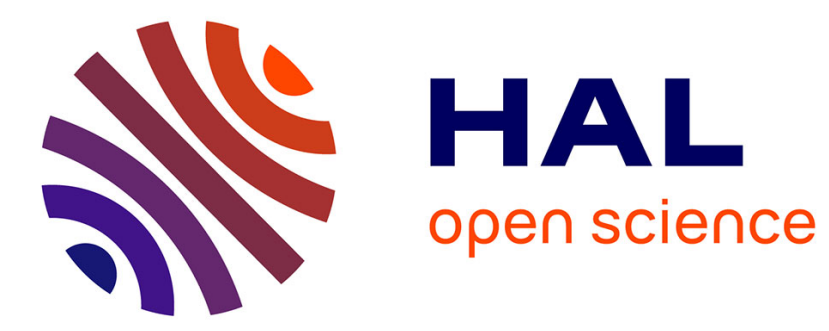

\title{
Les mesures en radioactivité et l'étalon du radium
}

Marie Curie

\section{To cite this version:}

Marie Curie. Les mesures en radioactivité et l'étalon du radium. J. Phys. Theor. Appl., 1912, 2 (1), pp.795-826. 10.1051/jphystap:019120020079500 . jpa-00241800

\section{HAL Id: jpa-00241800 https://hal.science/jpa-00241800}

Submitted on 1 Jan 1912

HAL is a multi-disciplinary open access archive for the deposit and dissemination of scientific research documents, whether they are published or not. The documents may come from teaching and research institutions in France or abroad, or from public or private research centers.
L'archive ouverte pluridisciplinaire HAL, est destinée au dépôt et à la diffusion de documents scientifiques de niveau recherche, publiés ou non, émanant des établissements d'enseignement et de recherche français ou étrangers, des laboratoires publics ou privés. 
Mme CURIE. MESURES EN RADIOACTIVITÉ ET ÉTALON DL RADIUM 793

\title{
LES MESURES EN RADIOACTIVITÉ ET L'ÉTALON DU RADIUM;
}

\author{
Par $\mathrm{I}^{\mathrm{m} \bullet} P$. CLRIE.
}

La radioactivité est une nouvelle science qui nous offre l'exemple d'un développement merveilleusement rapide. Rappelons en quelques mots les étapes principales de cette évolution.

Une nouvelle propriété de la matière est observée. Une série de recherches systématiques conduit à une première hypothèse fondamentale qui attribue à cette nouvelle propriété un caractère essentiellement atomique. En poursuivant les conséquences de cette hypothèse jusque dans l'étude de faits nouveaux, en apparence incompatibles avec elle (activité de certains minéraux), on est conduit à la découverte d'éléments chimiques nouveaux extrêmement rares qui deviennent un moyen d'action puissant dans l'exploration du nouveau domaine.

Des considérations d'ordre énergétique appliquées au phénomène atomique de la radioactivité et guidées par le nombre toujours croissant des faits observés font ensuite admettre une deuxième hypothèse fondamentale, d'après laquelle les atomes des éléments radioactifs sont instables et éprouvent des translormations successives. Cette hypothèse donne naissance à la théorie de désintégration atomique qui permet de classer d'une manière satisfaisante les phénomènes radioactifs connus. Comme confirmation, un fait indiscutable de transformation atomique est bientôt découvert (formation de l'élément hélium aux dépens de l'élément radium). On voit ainsi se fortifier la conviction que les phénomènes radioactifs nous donneront le moyen d'explorer la structure des atomes; ils sont actuellement les seuls qui donnent cet espoir, car seuls ils ont le caractère atomique fondamental sans aucune influence des liaisons moléculaires.

En même temps nous voyons s'accroître nos connaissances relativement au ròle des phénomènes radioactifs dans l'univers, et ce rôle apparaît de plus en plus important. Nous constatons que ces phénomènes sont très répandus dans la nature et qu'ils interviennent dans les interprétations qui peuvent ou pourront être données en ce qui concerne la formation des atomes, la succession des espèces minérales, les conditions thermiques et électriques de la terre et, plus généralement, du système solaire, l'état d'électrisation de l'atmo- 
sphère. Leur intluence se manifeste de plus dans divers phénomènes physiologiques, tels que le développement et la croissance des animaux et des végétaux. Ils apparaissent de plus en plus comme un des facteurs importants dans l'évolution de l'univers.

Indépendamment de cette haute importance dans le domaine de la science pure, les phénomènes radioactifs prennent chaque jour une extension croissante dans le domaine des applications médicales. Grands sont déjà les bienfaits des substances radioactives vis-à-vis de la souffrance humaine, et ce n'est certes pas une des moindres raisons de leur importance.

Ayant ainsi retracé les résultats de l'évolution admirable accomplie en quinze ans, je crois utile d'attirer l'attention sur le rôle des mesures precises dans la marche de cette évolution, rôle particulièrement frappant dans le cas que nous envisageons.

L'une des propriétés les plus importantes des corps radioactifs consiste à rendre conducteur l'air dans leur voisinage. La conductibilité est due à l'ionisation del'air par les rayons émis par ces corps. Considérons une enceinte fermée ou chambre d'ionisation occupée par l'air ainsi ionisé. Au moyen d'une électrode isolée plongeant dans la chambre, on peut établir dans celle-ci un champ électrique, et, en donnant au champ une intensité convenable, on peut recueillir sur les deux électrodes (paroi interne de la chambre et conducteur isolé), le total des quantités d'électricité égales et de signes contraires portées par les ions positifs et négatifs produits dans la chambre, chaque électrode recueillant les ions dont le signe est contraire au sien, et le nombre d'ions positifs et négatifs qui ont pu se recombiner entre eux avant d'avoir été recueillis pouvant être tout à fait négligeable. Si la cause de production d'ions agit d'une manière continue (rayonnement d'une substance radioactive), chaque électrode recueille de l'électricité d'une manière continue, de sorte qu'un courant électrique continu traverse la chambre d'ionisation. Le courant obtenu dans les conditions indiquées ci-dessus se nomme courant de saturation et peut fournir une mesure bien définie de l'intensité du rayonnement dans la chambre. La substance radioactive qui produit le rayonnement peut ètre placée à l'intérieur de la chambre; elle peut aussi se trouver à l'extérieur de celle-ci et agit alors grâce 
LES MESURES EN RADIOACTIVITÉ ET L'ÉTALON DU RADIUM 797

à l'émission de rayons qui traversent les parois (rayons pénétrants).

Dès le début des études concernant la radioactivité des composés d'urane et la recherche de la propriété radioactive dans d'autres substances, une méthode de mesures précises a été utilisée pour évaluer le degré de radioactivité par le courant de saturation produit dans des conditions déterminées. Les intensités très faibles des courants dont il s'agissait étaient mesurées. au moyen d'un dispositif comprenant un électromètre et un quartz piezoélectrique. Ce dispositif, qui a été fréquemment décrit (1) et qui est devenu classique, permet d'obtenir avec une grande exactitude la valeur absolue d'un courant de l'ordre de $10^{-12}$ a $10^{-10}$ ampère ; il permet aussi d'excellentes comparaisons en valeur relative. L'emploi d'une méthode aussi sûre a permis de faire un grand nombre de déterminations numériques, qui ont conduit à établir tout d'abord que la radioactivité des composés d'urane et de thorium est une propriété atomique des éléments uranium et thorium. La même mèthode a ensuite servi à mettre en évidence les activités, en apparence anormales, des minéraux, qui sont du même ordre de grandeur que celles des composés d'uranium, de sorte que la différence aurait pu échapper à des procédés d'observation grossiers. Ce sont cependant justement les observations faites à ce sujet qui ont conduit à la découverte du radium. Enfin, pendant le travail laborieux qui a dû être fait pour démontrer l'existence des éléments nouveaux, en particulier du radium, c'est toujours encore cette même méthode qui a été utilisée et l'on peut dire que sans son secours le travail n'aurait pu être mené à bonne fin. Ce n'est que grâce aux indications quantitatives précises qu'il a été possible de suivre les substances actives lors des séparations chimiques, de manière à rassembler finalement les traces de ces substances contenues dans des tonnes de matière première.

Le travail relatif à la découverte du polonium et du radium a été le premier travail chimique effectué à l'aide d'une méthode de mesures électrométriques. Mais l'emploi d'une telle méthode s'est ensuite généralisé et a permis d'effectuer un grand nombre d'autres travaux importants, tels que la séparation et l'étude de plusieurs corps radioactifs nouveaux solides et gazeux (actinium, radiothorium, mésothorium, radioplomb, ionium, émanations radioactives),

(1) M. Curie, Rev. gén. des Sc., janvier 1899; Thèse de doctorat, Paris, 1903 ; Traité de Radioactivité, 1910. 
les recherches qui ont prouvé la formation de radium dans les minerais d'urane ou celles qui consistent à compter les rayons de nature corpusculaire émis par les corps radioactifs (numération des particules $\alpha$ ), etc. Ainsi la technique électrométrique est devenue pour la radioactivité l'instrument de recherches le plus précieux, auquel cette science doit ses plus beaux triomphes - et dont elle espère d'autres dans l'avenir.

On conçoit d'après cela combien il est important de ne rien négliger de ce qui peut rendre l'usage des méthodes de mesures électrométriques en radioactivité encore plus sûr et plus facile.

Je suis ainsi amenée à expliquer comment, depuis plusieurs années, les savants qui s'occupent de radioactivité se sont formé la conviction toujours grandissante de la nécessité de constituer un étalon international de radium, et de réaliser par ce moyen un perfectionnement important, destiné à apporter plus de clarté et de sûreté dans l'activité des laboratoires intéressés.

Pour faire comprendre la signification d'un tel étalon et l'importance qu'il pourra avoir, il est nécessaire d'examiner plus en détail la technique de ces laboratoires.

Le caractère fondamental des méthodes utilisées en radioactivité est de devoir s'appliquer à l'étude de substances présentes en quantité infinitésimale. Cela est aussi vrai pour les méthodes chimiques que pour les méthodes physiques. Parmi les corps notablement radioactifs, seuls l'uranium et le thorium se trouvent dans leurs minéraux en proportion importante. L'activité de ces corps est faible et le champ des recherches qu'ils permettent d'effectuer est par là limité.

Le corps qui constitue l'instrument de recherches le plus important est le radium. Il doit son importance au concours de plusieurs circonstances. Tout d'abord son activité spécifique est considérable. Un gramme de radium qui vient d'être séparé du minerai a une activité environ 1,3 $10^{6}$ fois plus grande que celle d'un gramme d'uranium. La théorie des transformations radioactives nous apprend qu'il en est ainsi parce que le radium est un élément qui se transforme beaucoup plus rapidement que l'uranium. Toute substance radioactive simple se détruit suivant une loi dite exponentielle, d'après la- 
LES MESURES EN RADIOACTIVITÉ ET L'ÉTALON DL RADILM 799

quelle le temps nécessaire pour que la moitié de la substance subisse la transformation est une constante indépendante de la quantité actuellement présente; cette constante nommée période constitue un caractère fondamental de la substance. On !peut aussi définir une autre constante nommée vie moyenne et représentant pour un grand nombre d'atories d'une espèce déterminée la valeur moyenne des vies de ces atomes. La vie moyenne est en relation simple avec la période, elle varie proportionnellement à celle-ci. L'uranium et le radium proprement dit ou radium au minimum d'activité émettent chacun un rayonnement de l'espèce $\alpha$ ou rayonnement corpusculaire à charge positive. On peut montrer que les vies moyennes des deux corps doivent être entre elles approximativement comme les activités spécifiques, et il en sera de même chaque fois que l'on comparera deux corps radioactif́s à rayonnement analogue (1). La vie moyenne du radium a pu être déterminée par des expériences indirectes, elle est d'environ 2800 ans; celle de l'uranium doit être de l'ordre de $10^{9}$ ans.

Ce n'est pas seulement par son rayonnement propre que le radium est précieux. Ce corps est le siège de transformations en vertu desquelles il est généralement accompagné par un certain nombre d'autres substances radioactives. L'atome de radium, en se transformant, produit en premier lieu un atome d'hélium ou particule $\alpha$ émise avec grande vitesse et constituant un rayon $\alpha$, en second lieu un atome d'un gaz radioactif nommé émanation du radium. Ce gaz, dont la période est de $\mathbf{3 , 8 5}$ jours, se transforme à son tour avec émission de rayons $\alpha$ et formation d'une série de substances solides nommées radium $\mathrm{A}, \mathrm{B}, \mathrm{C}, \mathrm{D}, \mathrm{E}$ et $\mathrm{F}$, dont chacune résulte de la transformation de la précédente. Les rayonnements qui accompagnent chacune de ces transformations sont différents. Les corps A et F émettent des rayons $\alpha$, les corps $B$ et $D$ émettent des rayons $\beta$ ou électrons négatifs de grande vitesse, le corps $E$ émet des rayons $\beta$ et aussi des rayons $\gamma$ de nature probablement électromagnétique; enfin le corps $\mathrm{C}$, probablement complexe, émet des rayons $\alpha$, des rayons $\beta$ de vitesses variées s'approchant de la vitesse de la lumière et des rayons $\gamma$ extrêmement pénétrants.

Les vies moyennes des substances $\mathrm{A}, \mathrm{B}$ et $\mathrm{C}$ sont relativement

(1) La vie moyenne de l'uranium est probablement $2 \times 1,310^{\circ}$ fois plus grande que celle du radium, parce que, par atome transformé, deux rayons $\alpha$ sont émis dans le cas de luranium et un seulement dans le cas du radium. 
courtes (entre 4 minutes et 40 minutes); l'ensemble de ces substances constitue le dépôt actif à érolution rapide; les vies moyennes des corps D, E et F sont beaucoup plus longues (entre six mois et vingt ans); ces corps forment le dépòt actif à évolution lente. Voici le schéma des transformations du radium ( $f g .1)$.

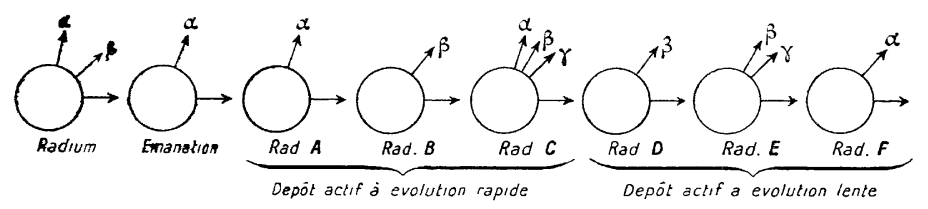

FIG. 1.

Quand le radium est à l'état solide, dans un sel ou dans un minerai, l'emanation du radium reste entièrement ou presque entièrement occluse dans la matière solide et se iransforme sur place en matières solides qui s'accumulent. Mais l'accumulation étant limitée par la destruction spontanée, il s'établit un état de régime dans lequel la production pour chaque matière compense la destruction. Le radium est alors à l'état d'équilibre de régıme radioactif avec ses dérivés. Un tel état d'équılıbre peut s'établir et persister, en apparence invariable, pendant des années, parce que la vie moyenne du radium est très longue par rapport à une année et par rapport aux vies moyennes de tous les derivés. Un premier équilibre avec l'émanation et le dépût actif à évolution rapide est atteint en un mois environ à partir du moment où le radium est mis en un état dans lequel l'émanation ne peut s'échapper au dehors; cette condition est réalisée rigoureusement quand le radium, dans n'importe quel état, est enfermé en tube scellé; elle est réalisée approximativement pour un sel solide calciné. En vertu de cette évolution, l'activité du radium solide va en augmentant et devient $\mathbf{b}$ à 6 fois plus elevée que la valeur minimum. En n ême temps la variété du rayonnement augmente et l'on voit, en particulier, apparaître des rayons très penétrants qui n'appartiennent pas au radium élément, mais à ses dérivés. C'est à cet état que le radium est généralement utilisé pour les recherches ou pour les applications médicales.

Quand cette première évolution est achevée, elle en laisse subsister une autre, beaucoup plus lente, qui consiste dans l'accumulation du dépôt actif à évolution lente $t$ tend vers un équilibre radioactif avec celuı-ci. Cet équllibre demande environ cent ans pour s'etablir, 
LES MESURES EN RADIOAGTIVITÉ ET LÉTALON DL RADILM 801 et l'on peut prévoir qu'à la limite l'activité totale du sel solide serait encore augmentée d'environ 20 0/0. Cette limite représente un maximum suivi d'une décroissance extrèmement lente du rayonnement, suivant la loi de transformation du radium, la diminution par année étant inférieure à 1 p. 1000.

Les minéraux dans lesquels on trouve le radium sont des minéraux d'urane. Leur âge depuis la formation est de l'ordre de cent millions d'années. La présence de radiuı dans des minéraux de formation aussi ancienne ne peut être expliquée que par l'hypothèse d'une production sur place. Il semble établi, en effet, que le radium est un dérivé de l'uranium et se trouve en équilibre avec celui-ci dans les minerais d'urane les plus anciens. La vie moyenne de l'uranium est de l'ordre de la durée des époques géologiques. Dans certains minéraux qui ne dégagent sensiblement pas d'émanation, l'uranium se trouve en équilibre radioactif avec le radium et tous les dérivés de celui-ci. Il a été prouvé que le dernier de ces dérıvés ou radium $\mathrm{F}$ est identique au polonım, qui est la première substance nouvelle fortement radioactive truuvée dans les minerais d'urane.

La proportion de radium dans les minéraux d'urane offre un rapport approximativement constant avec la proportion d'urane, de sorte que l'on a environ $3,410^{-7}$ gramme de radium par gramme d'uranıum. Les minéraux riches en uranium (contenant, par exemple, $750 / 0$ d'oxyde d'uranium) renferment par tonne de minerai quelques décigrammes de radium. Les méthodes d'investigation chimique et même l'analyse spectrale eussent été incapables de déceler la presence de ce corps, qui pourtant a un spectre très sensible, et c'est seulement grâce à la propriété radioactive, mise en évidence par une methode électrométrique, que la découverte a pu être faite. On sait maintenant que la réaction radioactıve du radium est plusieurs milliers de lois plus sensible que sa reaction spectrale, laquelle est du même ordre de sensibilité que celle du baryum.

Bien que la proportion de radıum dans les minerais d'urane soit très laible, l'extraction de ce corps et la préparation de sels purs a pu être effectuée avec succès. Le radium est un élément alculino-terreux, dont le poids atomique est égal à $226, \breve{3}$. Grâce à ses propriétés chimıques très caractérisées, sa préparation, déjà très bien etudiée, ne présente pas de difficultés très grandes. Cette préparation fait maintenant l'objet d'une industrie qui occupe plusieurs usines en France et à l'étranger. Mais, par suite de la rareté des minerais, du 
travail laborieux de l'extraction et des besoins croissants, en vue d'applications scientifiques et médicales, le prix de venie est extrêmement élevé. Le radium est la plus coûteuse de beaucoup de toutês les substances connues. Le prix actuel est de $\$ 00000$ francs le gramme de bromure de radium. La quantité totale de radium jusqu'ici extrait du minerai n'atteint probablement pas 10 grammes.

Si lon envisage les substances fortement radioactives autres que le radium (actinium, polonium, mésothorium, radiothorium), on constate que plusieurs d'entre elles peuvent avoir un rayonnement spécifique plus intense même que celui du radium. On a pu préparer des échantillons de polonium et de mésothorium accompagné de radiothorium dont l'activité était de mème ordre que celle du radium à poids égal, alors que cependant la substance active n'était présente qu'en proportion très faible. Aucune de ces substances n'a, en effet, encore pu être isolée. La préparation de ces corps est laborieuse et leur proportion dans la matière première est considérablement plus faible encore que dans le cas du radium. Cela tient à ce que leurs vies moyennes sont courtes par rapport à celle du radium (polonium, 200 jours ; actinium, trente ans ? mésothorium, environ huit ans ; radıothorium, environ trois ans). 11 en résulte aussi que les produits preparés à grand'peine et à grands frais ne donnent pas, comme le radıum, le benéfice d'une invariabilité suftisante au point de vue pratique, mais perdent leur valeur en quelques annees. La préparation de matières très actives dont l'activité est due au mésothorıum accompagné de radiothorium et de ses dérivés, est cependant devenue industrielle, car la matière première (minerais et sels de thorium) se trouve disponible en assez grande abondance. Le rayonnement des matières ainsi obtenues est aussi riche et aussi varie que celui du radium et peut rendre des services analogues; mais il a l'inconvénient d'évoluer avec le temps suivant une loi compliquee.

L'ionium a une activité spécitique plus petite que celle du radium. Ce corps doit se trouver dans les minerais en proportion plus forte que le radium, mais sa préparation semble difficile. Son rayonnement oflre moins de variété que celui du radium.

En résumé, on ne peut refuser au radium un rôle prépondérant qu'il semble destiné à conserver. 11 en est redevable : $1^{\circ}$ à la valeur appropriée de sa vie moyenne,qui n'est ni trop grande ni trop petite et lui assigne une grande activité avec une stabilité suffisante; $2^{\circ}$ à ses proprietés chimiques qui permettent de le séparer sans trop de 
LES MESURES EX RADIOACTIVITÉ ET LÉTALON DL RAHLY 803 peine $; 3^{\circ}$ à la richesse de son rayonnement dû en partie au radium même, en partie aux dérivés à évolution rapide $; 4^{\circ}$ à la rapidité de la première évolution qui assure, un mois après la préparation, un rayonnement approximativement constant pendant des anuées ; $5^{\circ}$ à ce fait important que le radium a pu être isolé et bien défini comme élément chimique et qu'ainsi il offre une base solide pour des recherches quantitatives.

Ainsi s'explique ce fait que la vraie technique de précision des laboratoires de radioactivité est basée sur l'emploi du radium. C'est donc de ce corps qu'il sera principalement question dans la suite.

Par suite de l'extrème dilution des corps fortement radioactifs dans la matière première, les réactions chimiques utilisées pour la séparation doivent ètre considérées d'une manière spéciale. Dans le traitement de minerais, la solubilité des composés de corps radioactifs devrait toujours ètre suffisante pour empècher leur précipitation ou cristallisation. Celle-ci n’a lieu que grâce à la présence de sels isomorphes (cristallisation de sels de baryum radifères) ou de sels qui en précipitant, ont la faculté d'entrainer certaines substances actives (entrainement d'actinium ou de mésothorium parle sulfate de baryum), ou encore de substances insolubles qui retiennent les matières actives par adsorption (action du charbon pour recueillir dans une solution l'uranium \% ou d'autres substances). Ces réactions d'entraînement peuvent ètre différentes de celles que les substances presenteront à l'état de concentration moléculaire normale. Il est d'alleurs tout naturel, au point de vue des theories moléculaires, que les propriétés d'une matiere puissent se moditier quand chacune de ses molécules se trouve isolée au sein d'une matière étrangère. Il est remarquable, par contre, que la propriété radioactive ne semble pas intervenir pour créer des propriétés moléculaires spéciales. Ainsi les réactıons du radium au point de vue moléculaire n'offrent aucune singularité ; elles correspondent, ainsi que l'aspect du spectre, à la position qui est assignée au radium par son poids atomique dans la classitication périodique des éléments.

Examinons maintenant les conditions de l'emploi du radium au point de vue quantitatif. Eu égard à la rareté de cette substance, à l'insuffisance de la production et au prix considérable, la quantité la plus forte de cette matière réunie dans une des institutions existantes (laboratoires ou instituts scientifiques ou médicaux) est de l'ordre du gramme; le plus souvent cette quantité est très inférieure. La quan- 
tité de radium utilisée ponr les travaux scientifiques des laboratoires ne dépasse donc pas actuellement la limite indiquée et reste, en règle générale, très au-dessous. Pour un grand nombre de travaux, des quantités variant du centigramme à une fraction de milligramme sont d'usage courant. Il est bien évident qu'il ne saurait être question de doser ces petites quantités avec précision au moyen d'une balance. La pesée des sels de radium est d'ailleurs une opération délicate, demandant de grandes précautions, parce queces substances absorbent la vapeur d'eau de l'atmosphère et parce qu'elles éprouvent des transformations chimiques moléculaires sous l'influence de leur propre radiation, de sorte que la nature du sel n'est bien définie qu'immédiatement après la préparation.

ll existe toute une série de travaux scientifiques où les quantités de radium qui entrent en jeu sont encore beaucoup plus faibles que celles indiquées ci-dessus. Ce sunt les travaux relatifs à la radioactivité du sol, des eaux et de l'atmosphère. On a pu prouver qu'à l'état de dilution extrême le radium est répandu partout à la surface de la terre, dans les roches superficielles, dans l'eau des mers, dans les eaux des sources. La proportion de radium par gramme de ces substances est de l'ordre de $10^{-12}$ gramme ou au-dessous, rarement audessus dans quelques minéraux, eaux minérales ou petroles naturels. Les quantités de matière sur lesquelles on opère étant généralement inférieures à 1 kilıgramme, on est donc là en présence d'un problème qui consiste à doser avec précision une quantité de radium de l'ordre de $10^{-10}$ gramme. Ce problème, absolument inabordable pour l'analyse chimique quantitative, a été résolu par l'emploi de méthodes electrométriques.

L'almosphère ne contient pas de radium, mais contient de l'émanation du radium à l'état de traces. Plusieurs eaux minérales contiennent de l'emanation sans contenir du radium. Dans un grand nombre de travaux de liaboratoire on utilise comme source de rayons l'émanation du radium séparee de celui-ci.

Pour pouvoir faire du travail quantitatif, il a fallu établir des méthodes permettant de doser l'émanation. On verra d'ailleurs l'usage de ces méthodes pour le dosage mème du radium. En raison de son activité spécitique bien plus elevée que celle du radium, l'émanation a une réaction radioactive environ 100000 fois plus sen-ible. En revanche, elle n'est jamais présente qu'en quantités extrêmement faibles, et l'on ne pourrait songer à la mesurer ni au poids ni au 
LES MESURES EN RADIOACTIVITÉ ET LÉTALON DL RADIUM 80ว volume. La plus grande quantité que l'on puisse songer à isoler dans un des laboratoires les plus riches en radium est de l'ordre de 1 millimètre cube. Par la méthode électrométrique on peut doser $10^{-10}$ millimètre cube d'émanation.

Il ne sera pas question ici de dosage des dérivés suivants du radium, bien que les substances $\mathrm{A}, \mathrm{B}$ et $\mathrm{C}$ interviennent dans les mesures ainsi que nous le verrons dans la suite. Je ferai remarquer seulement que ces substances, en raison de leurs vies très brèves, ne peuvent être présentes qu'en quantité encore considérablement plus faible que l'émanation du radium. Par contre la sensibilité de leur réaction radioactive devient énorme. Ces substances peuvent être obtenues par dépôt spontané sur un fil métallique, de preférence chargé négativement et exposé dans un espace contenant de l'émanation du radium. On peut alors les traiter comme des substances chimiques et les faire passer dans une dissolution acide. D'une telle dissolution on peut les reprécipiter sur un métal approprié, ou par entraînement avec un sel dont on eflectue la précipitation. Toutes ces opérations peuvent être suivies par le moyen de dosages électrométriques. Il est tout à fait curieux de voir qu'on peut ainsi traiter par des méthodes chimiques et doser avec précision des matières qui, selon toute vraisemblance, ne pourront jamais être caractérisées commı éléments chimiques, leur destruction étant trop rapide. C'est là une nouvelle chimie qui utilise comme outil principal l'électromètre et non la balance, et que l'on est tenté de nommer la chimie de l'invisible.

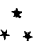

La méthode tout d'abord utilisée pour le dosage approximatif du radium consistait à mesurer le courant de saturation produit dans une chambre d'ionisation par une certaine quantité de la substance radifère solide, pulvérisée et étalée en couche mince sur un plateau placé dans la chambre. Un dispositif très fréquemment utilisé à cet effet a été décrit en plusieurs circonstances (1). Cette méthode utilise le rayonnement total de la substance; elle est, pour cette raison, très sensible et permet par exemple, de mesurer facilement l'activité

(1) M. Curie, Rev. gén. des Sciences, janvier 1899: Thèse de doctorat, Paris, 1903. 
d'une substance contenant le radium dans la proportion d'un dix-millionième. Par l'usage de surfaces variées on peut faire varier la sensibilité de la méthode et l'adapter à des concentrations qui varient dans de larges limites. Le rayonnement utilisé dans ces mesures est presque uniquement le rayonnement $\alpha$ qui est très absorbable, de sorte quà partir d'une faible épaisseur de la couche active le courant n:augmente plus guère avec l'épaisseur. Les couches utilisées en pratique peuvent avoir $0^{\mathrm{mm}} . \ddot{\Xi}$ d'épaisseur, et elles pourraient être plus minces si elles étaient très continues.

Cette méthode a rendu et rend toujours de très grands services pour les déterminations approchées de la richesse en radium et pour le contrôle de lextraction de cette substance du minerai. Cependant elle n'a pas été élaborée de manière à devenir une méthode de précision. En voici les raisons:

$1^{\circ}$ Elle nécessite une manipulation de la substance qui présente de grands inconvénients quand il s'agit de matières riches en radium et par conséquent très coûteuses.

$2^{\circ}$ Elle n'indique pas la quantité totale de radium, mais la concentration en radium. Encore celle-ci n’est-elle donnée qu'avec une certaine approximation, parce que la matière inactive qui accompagne le radium exerce sur les rayons qui la traversent une absorption qui dépend de la nature de cette matière.

$3^{\circ}$ L'état des matières radifères solides à l'air libre n'est pas très bien défini. Ces matières peuvent dégager plus ou muins d'emanation, de sorte que leur activité dépend des quantités d'émanation et de dépôt actif contenues dans le sel. L'activité la mieux définie est l'activité minimum que le sel possède quand il ne contient ni émanation ni dépôt actif ; on réalise cet état en dissolvant le sel et en chassant l'émanation, et en évaporant ensuite le sel à sec avec des précautions convenables. Dans bien des cas, ces opérations ne sont pas admissibles; elles ne peuvent, en particulier, être appliquées aux petites quantités de matières précieuses.

Nous possédons deux méthodes précises qui permettent de doser le radium et son émanation : $\mathbf{1}^{\circ}$ mesure direcle de l'émanation et dosage du radium par l'émanation dégagée; $2^{\circ}$ mesure du radium ou de l'émanation du radium par l'émission de rayons pénétrants (rayons $\gamma$ ).

Mesures directes d'émanation. - L'émanation du radium étant introduite dans une chambre d'ionisation, on peut mesurer le courant 


\section{LES MESURES EN RADIOACTIVITÉ ET L'ÉTALON DU RADILM 807}

de saturation obtenu. Ce courant subit une certaine évolution avec le temps. Il augmente d'abord par suite de la formation du dépôt actif aux dépens de l'émanation, atteint un maximum en 3 heures et entre ensuite en décroissance suivant la loi exponentielle caractéristique de l'émanation.

Cette loi peut s'écrire:

$$
q=q_{0} e^{-\frac{t}{\sigma}},
$$

où $q$ et $q_{0}$ sont les quantités d'émanation présentes respectivement aux temps $t$ et 0 et $\theta$ est la vie moyenne de l'émanation : celle-ci est égale à 133 heures et correspond à une période de 3,8 jours.

La mesure du courant peut être faite avec une grande précision pendant l'état stationnaire qui correspond au maximum. On utilise le rayonnement total de l'émanation et du dépòt actif, on dispose donc d'une grande sensibilité.

L'émanation est produite par le radium d'une manière continue avec un débit constant, proportionnel à la quantité de radium et indépendant de l'état de combinaison moléculaire. Si la production a lieu en vase clos, la quantité d'émanation augmente avec le temps et tend vers une valeur limite $q_{\infty}$ qui ne dépend que de la quantité de radium présente dans le vase et lui est proportionnelle.

Si à l'origine du temps le vase ne contenait pas d'émanation, l'accumulation de celle-ci en fonction du temps $t$ a lieu suivant la loi :

$$
q=q_{\infty}\left(1-e^{-\frac{t}{i}}\right),
$$

où $\theta$ est la vie moyenne de l'émanation.

Pour doser le radium en valeur relative, on procède de la manière suivante: la matière radifère est mise en solution et introduite dans un vase; on chasse l'émanation contenue dans le vase en faisant bouillir la solution ou en la laissant traverser par un courant d'air ; ensuite on ferme le vase et on laisse l'émanation s'accumuler pendant quelques jours. Après ce temps l'on procède à l'extraction de l'émanation accumulée et l'on transporte celle-ci dans une chambre d'ionisation appropriée, afin de mesurer après 3 heures le courant de saturation à son maximum qui donne une mesure de $q$. La valeur de $q_{\infty}$ peut alors ètre calculée par l'emploi de la formule théorique (2) 
et donne une mesure de la quantité de radium dans le vase. Pour effectuer les calculs on se sert de tables numériques spécialement construites et utilisant la constante $\theta$ très exactement déterminée par l'expérience.

Pour rendre la mesure absolue, il est nécessaire de déterminer le courant de saturation maximum pouvānt être produit dans une chambre d'ionisation donnée par la quantité d'émanation limite qui correspond à une quantité connue de radium. Cette opération très délicate exige la pesée d'une certaine quantité d'un sel de radium parfaitement défini que l'on utilise pour préparer une solution titrèe devant servir pour l'étalonnage de l'appareil de mesures.

Le même appareil pourra servir pour la mesure de quantités d'émanation, indépendamment de leur provenance, par le courant de saturation maximum qu'elles peuvent produire. Toute quantité d'émanation pourra ainsi être égalée à la quantité limite $q_{\infty}$ qui serait en équilibre radioactif avec une quantité connue de radium.

Le courant de saturation qu'on mesure varie avec la forme et les dimensions de la chambre d'ionisation et augmente avec le volume de celle-ci, de sorte qu'un étalonnage n'a de valeur que pour une chambre déterminée. Si cependant les dimensions de celle-ci sont exactement indiquées et si le courant obtenu avec l'émanation limite d'une quantité connue de radium est donné en unités absolues, l'appareil de mesures pourra être reproduit dans un laboratoire qui pourra s'en servir pour des mesures absolues sans posséder de solution étalon. On peut aussi utiliser une formule empirique qui permet de ramener le courant observé à la valeur qu'il aurait eu si la chambre d'ionisation avait un volume infini ( $\left.{ }^{1}\right)$.

Voici l'appareil utilisé pour ce genre de mesures dans le Laboratoire de Radioactivité de Paris.

La boîte cylindrique $\mathrm{P}(f g .2)$ reliée à une batterie de charge à haut voltage, sert de chambre d'ionisation; une électrode isolée $\mathrm{E}$, reliée à l'électromètre, pénètre dans la chambre. Celle-ci est étanche, et munie de robinets qui permettent l'entrée et la sortie de l'émanation. La boîte T, reliée au sol, sert d'enveloppe protectrice.

Le courant produit par l'émanation augmente avec la masse d'air contenue dans la chambre. On doit donc veiller à ce que celle-ci soit la même dans chaque mesure, ou ce qui est plus facile, remplir la

(1) Duane et Labohide, Le Radium, 1910. 
LES MESURES EN RADIOACTIVITÉ ET L'ÉTALON DU RADIUM 809 chambre avec de l'air à la pression et à la température ambiante et effectuer une correction pour ramener les nombres obtenus à une masse d'air normale.

Le dosage du radium par la mesure de l'émanation dégagée est

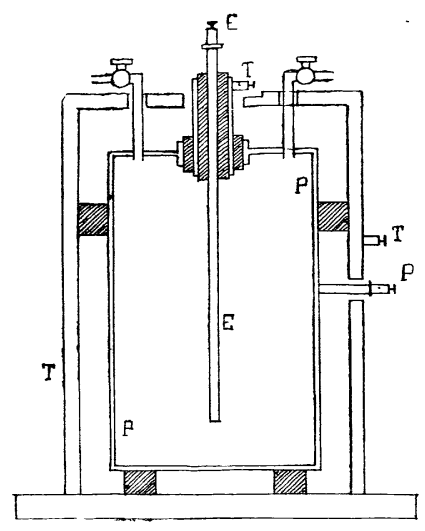

FIG. 2.

devenu une opération tout à fait courante dans la technique des laboratoires de radioactivité. Le dosage de l'émanation seule par ce procédé est surtout appliqué à l'étude de la teneur en émanation des eaux minérales.

La méthode qui vient d'être indiquée est extrêmement sensible. Elle permet de déceler $10^{-10}$ gramme de radium ou $10^{-10}$ millimètre cube d'émanation et même moins. Elle permet d'effectuer des mesures relatives avec une précision de $0, \breve{0} 0 / 0$ pour des quantités de radium de l'ordre de $10^{-8}$ gramme. Mais la valeur absolue des mesures dépend essentiellement de l'exactitude de l'étalonnage de l'appareil de mesures, c'est-à-dire, en dernier lieu, de la connaissance exacte de la quantité de radium contenue dans la solution étalon.

Mesures de rayons $\gamma$. - Le radium en équilibre radioactif avec l'émanation et le dépôt actif à évolution rapide, émet un rayonnement qui comprend des rayons extrêmement pénétrants nommés rayons $\gamma$ et pouvant traverser plusieurs centimètres de métal. Le pouvoir pénétrant de ces rayons est tel que, pour réduire leur intensité de moitié, il faut interposer sur leur trajet une épaisseur de plomb d'environ 2 centimètres ou une épaisseur plus grande encore de toute matière 
moins dense. Quand la source radiante est entourée d'une enveloppe de plomb de quelques millimètres d'épaisseur, les rayons $\gamma$ seuls traversent l'enveloppe, tandis que les rayons $\alpha$ et les rayons $\beta$ sont absorbés par le plomb. Les rayons $\gamma$ ne constituent qu'une faible fraction du rayonnement total mesuré par lionisation produite.

Les rayons y n'appartiennent ni au radium élément ni à l'émanation; ils sont émis par le constituant $\mathrm{C}$ du dépôt actif à évolution rapide; leur intensité est proportionnelle à la quantité de Ra C. Quand le radium, après avoir été ramené au minimum d'activité, est enfermé en tube scellé, le rayonnement $\gamma$, d'abord nul, croit avec le temps et tend vers une valeur limite proportionnelle à la quantité de radium et à la quantité limite d'émanation. Après un jour la loi d'augmentation est de la forme:

$$
i=i_{\infty}\left(1-e^{\left.-\frac{t}{0}\right)}\right.
$$

où $i_{\alpha}$ représente lintensité limite du rayonnement, $i$ celle au temps $t$ et $\theta$ la vie moyenne de l'émanation. La limite est sensiblement atteinte en un mois.

De même, quand une quantité d'émanation vient d'ètre enfermée en tube scellé, le rayonnement $\gamma$, d'abord nul, croît avec le temps, atteint un maximum en 3 heures et décroìt ensuite suivant la loi de destruction de l'émanation, conformément à la formule :

$$
i=i_{0} e^{-\frac{t}{\theta}}
$$

Quand cette loi est établie, la proportion de radium C reste proportionnelle à la quantité d'émanation, et il en est de mème du rayonnement $\%$.

Il résulte de ce qui vient d'être dit que, pour des quantités de radium ou d'émanation de radium enfermées en tube scellé, la comparaison des rayonnements $\gamma$ émis permet une comparaison quantitative des quantités de matière active.

Dans le cas du radium, la mesure doit ètre faite quand l'équilibre radioactif est atteint, c'est-à-dire un mois environ après que l'échantillon a été enfermé en tube scellé à l'abri des pertes d'émanation (1).

(1) Au lieu d'attendre un mois, on peut effectuer, dès la première semaine, quelques mesures de l'accroissement avec le temps; on en deduit par un calcul simple la valeur du rayonnement limite. 


\section{LES MESLRES EN R.ADIOACTIVITÉ ET L'ÉTALON DL RADILY 811}

Le rayonnement \% émis mesure la quantité de radium métal présente dans la source radiante, à condition que l'absorption des rayons $\gamma$ dans la matière active et dans la paroi de l'enveloppe qui la renferme soit ou bien négligeable ou bien la même dans tous les cas. Quand il n'en est pas ainsi, il est nécessaire de tenir compte de cette absorption.

Les rayons utilisés sont ceux qui, pour pénétrer dans l'appareil

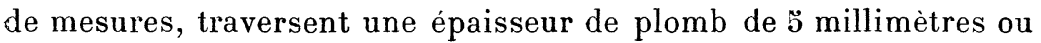
davantage. Ces rayons sont absorbés par la matière suivant une loi exponentielle. L'intensité du rayonnement décroît en fonction de l'épaisseur de la matière traversée suivant la loi

$$
i=i_{0} e^{-\because \cdot x}
$$

où $i_{0}$ est l'intensité initiale, $i$ celle qui reste après le passage au travers de l'épaisseur $x$ et $\mu$ un coefficient constant nommé coefficient d'absorption, dont la valeur dépend de la nature du corps ahsorbant et varie approximativement en raison inverse de la densité de celuici. Pour le plomb, on trouve $u=0,5 \frac{1}{\mathrm{~cm}}$; pour le verre, $\mu=0,1 \frac{1}{\mathrm{~cm}}$. Pour la matière du sel actif, la valeur de $\mu$ dépend de la nature du sel et de l'état de tassement; eu égard à la densité moyenne, on peut avoir approximativement un coefficient d'absorption moyen $\mu=0,05 \frac{1}{\mathrm{~cm}}$.

Les mesures précises ne peuvent faire intervenir l'absorption des rayons par la matière radioactive, car cette absorption n'est généralement pas assez bien définie. Pour cette raison, les dosages de précision par rayons $\gamma$ ne peuvent être effectués que sur de petites quantités de sels occupant très peu de volume et contenues généralement dans de petites ampoules en verre scellées. Il résulte de ce qui précède que, si la distance que les rayons ont à franchir dans la matière active est en moyenne de 1 millimètre, la fraction de rayons absorbés par la matière est de l'ordre de $0,30 / 0$ seulement et peut, le plus souvent, être négligée. Puisque les mesures de précision sont limitées aux petites quantités de matière, et que les rayons $\gamma$ ne constituent qu'une faible fraction du rayonnement total de la substance,il en résulte que le dosage du radium par les rayons $\gamma$ est loin d'être aussi sensible que le dosage approché par le rayonnement total ou que celui qui utilise la mesure de l'émanation dégagée. Le dosage de précision par rayons $\gamma$ s'applique facilement à la mesure de quantités 
de radium de lordre d'un milligramme. On peut considérer cette méthode comme au moins 1000 fois moins sensible que celle pa $\mathbf{r}$ l'émanation. En revanche, la mesure par rayons $\gamma$ offre cet avantage considérable qu'elle ne nécessite aucune manipulation dangereuse pour la sécurité des matières : elle permet d'effectuer la mesure sans retirer la matière du tube qui la contient. Cette méthode est, en particulier, la seule applicable au dosage commercial de petites quantités de sels de radium destinés à la vente. Diverses applications médicales utilisent précisément les rayons pénétrants. En ce cas, le dosage peut être fait sur l'ampoule mème qui sera mise en usage.

Pour des ampoules de verre dont les parois ont une épaisseur d'un demi-millimètre, la fraction de rayons $\gamma$ absorbée par le verre est d'environ $0,50 / 0$ et peut généralement être négligée. Quand le verre est plus épais, on se place dans les meilleures conditions en comparant des ampoules de même forme faites avec le même verre et produisant, par conséquent, la même absorption pour un remplissage analogue.

En ce qui concerne les dimensions des ampoules à comparer, il existe d'ailleurs des conditions géométriques indispensables pour assurer la précision des mesures. Le courant produit dans la chambre d'ionisation par les rayons issus de la source dépend de l'utilisation des rayons dans la chambre, etcelle-ci est déterminée par la position de la source par rapport à la chambre. Les conditions sont comparables quand des sources tout à fait semblables occupent successivement une position exactement la même par rapport à la chambre. Généralement il n'en sera pas ainsi et, pour chaque forme d'appareil de mesures, l'on devra se rendre compte dans quelle mesure on peut faire varier la forme, les dimensions et la position de la source, tout en conservant la précision voulue. On peut, en particulier, réaliser de bonnes conditions avec des sources dont les dimensions sont petites par rapport à celles de la chambre; en ce cas il est facile de donner à la source une position bien définie par rapport à la chambre. On peut obtenir ce même avantage en plaçant la source à grande distance de la chambre. mais ce procédé a l'inconvénient de diminuer encore grandement la sensibilité de la méthode.

Voici trois types dappareils qui servent pour les mesures de rayons $\gamma$ dans le Laboratoire de Radioactivité de Paris :

Lappareil $I$ ( fig. 3) se compose d'une boìte à paroi cylindrique $P$ 
LES MESLRE: EN RADIOACTIVITE ET LÉTALON DU RADILM 813
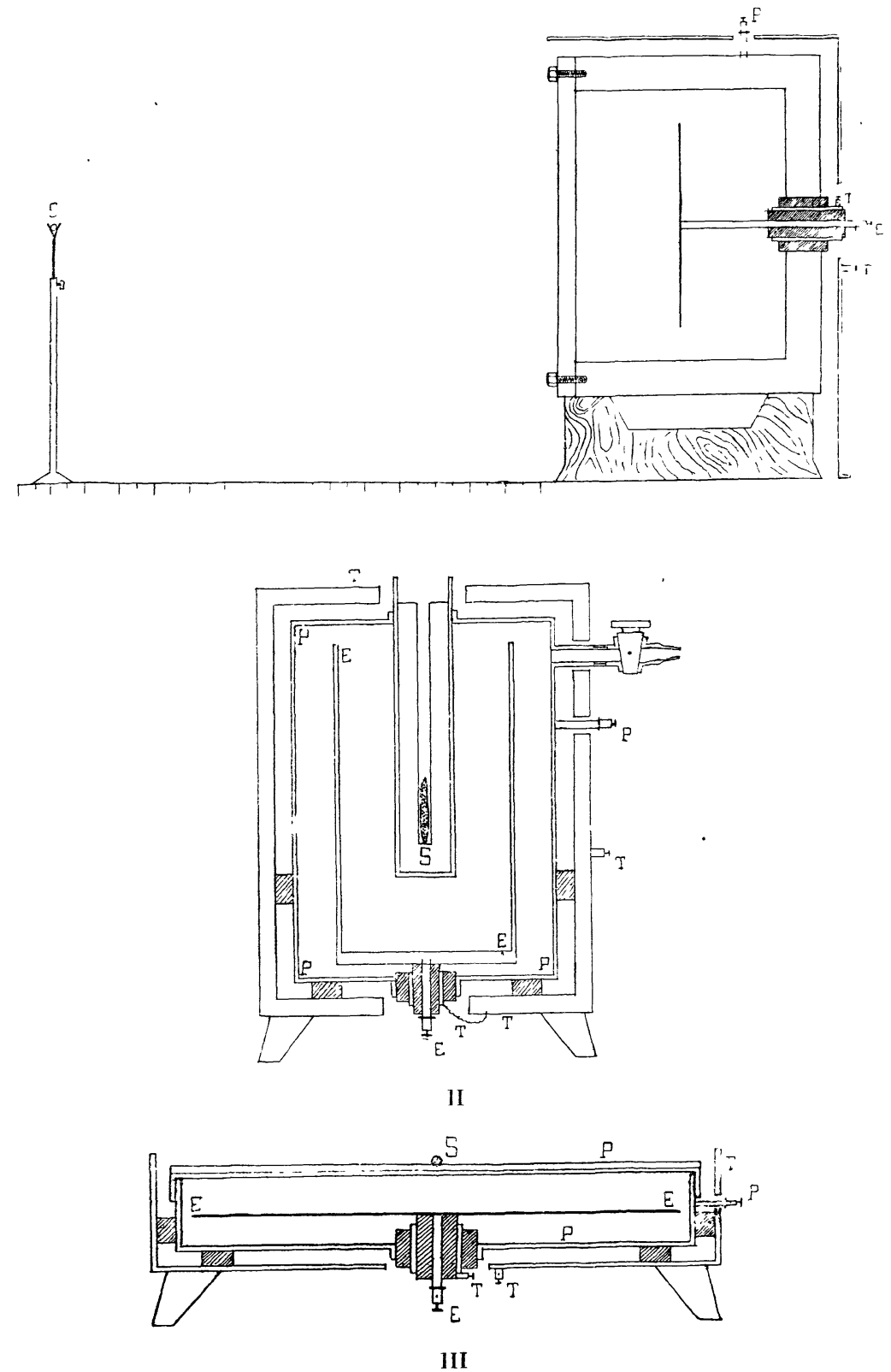

FIG. 3 . 
dans laquelle pénètre l'électrode isolée $\mathrm{E}$, reliée à l'électromètre. La boîte $\mathrm{P}$ est reliée à une batterie de charge à haut voltage. La source est placée en $\mathrm{S}$ à une distance convenable. Les rayons issus de la source ionisent l'air dans l'espace contenu à l’intérieur de la boîte $\mathrm{P}$ et formant chambre dionisation; les ions d'un signe produits dans cet espace sont recueillis par l'électrode E. L'écran T, relié au sol, sert d'enveloppe protectrice.

L'appareil II possède une électrode cylindrique $\mathrm{E}$, reliée à l'électromètre, et contenue dans une boîte cylindrique $P$, rcliée à la batterie. Un tube cylindrique à fond fermé, soudé à la boite $P$, pénètre à l'intérieur de la boìte $\mathrm{E}$. Dans ce tube est placée l'ampoule $\mathrm{S}$ qui contient le radium. L'électrode $\mathbf{E}$ recueille les ions formés dans l'espace intérieur à $\mathrm{E}$ et aussi ceux formés entre les surfaces $\mathrm{E}$ et $\mathrm{P}$; l'ensemble de ces deux espaces forme chambre d'ionisation. L'appareil est entouré d'une boîte protectrice T reliée au sol. Ce dispositif convient Irès bien pour la comparaison d'ampoules de formes peu différentes. La chambre d'ionisation peut être rendue complètement étanche, ce qui permet de travailler avec une masse d'air constante. Il y a à cela un avantage qui peut quelquefois être important, car le courant d'ionisation produit par un rayonnement donné, dépend de la densité de l'air contenu dans la chambre et augmente avec celle-ci.

L'appareil III est un grand condensateur à plateaux, composé du plateau $\mathrm{E}$ relié à l'électromètre et de la boîte cylindrique plate $\mathrm{P}$ reliée à la batterie de charge. La source est placée en $\mathrm{S}$ au centre de la paroi supérieure de la boîte $\mathrm{P}$. La boîte $\mathrm{T}$ reliée au sol protège lappareil. La chambre d'ionisation se compose des espaces compris entre le plateau isolé $\mathbf{E}$ et la boite $P$. Cet appareil comprend un grand volume d'air ionisé et offre une grande sensibilité. Il permet de comparer des ampoules de dimensions notablement différentes.

Dans tous les cas la mesure est obtenue par la différence des courants observés: $1^{\circ}$ quand l'ampoule est en place; $2^{\circ}$ quand elle est enlevée. L'air possède, en effet, une conductibilité propre qui peut être observée partout, mais qui est augmentée dans les laboratoires de radioactivité. Cette conductibilité croît avec le volume d'air dans la chambre. Les poussières contenues dans celle-ci étant entrainées vers les électrodes produisent aussi un courant assez irrégulier en l'absence de l'ampoule. Ces effets sont sensibles avec l'appareil III; on élimine les poussières en laissant l'appareil sous l'action du champ électrique quelque temps avant de procéder aux mesures. 
LES NESLRES EY RADIOACTIVITÉ ET L'ÉTALON DL RADILM 813

Pendant une longue série de mesures, la sensibilité d’appareils tels que III varie avec la pression et la température de l'atmosphère. On en tient compte par des mesures croisées ou des corrections appropriées.

Il existe un grand nombre de cas oì, au lieu d'utiliser une ampoule contenant du radium, on peut effectuer l'expérience avec une ainpoule contenant de l'émanation. On sait actuellement extraire l'émanation, la purifier et la refouler dans un très petit volume. La quantité d'émanation contenue dans l'ampoule peut convenablement être mesurée par le rayonnement $\gamma$. La mesure doit être faite au moins trois heures après la fermeture de l'ampoule. Eu égard à la décroissance de l'émanation, la mesure doit être rapportée à un instant assez bien déterminé. Pendant une heure l'activité décroît. de $0,760 / 0$.

L'ampoule ne contenant que du gaz, il n'y a pas lieu de s inquiéter de l'absorption des rayons à l'intérieur de l'ampoule. Quant à l'absorption produite par la paroi du tube, elle intervient comme dans le cas du radium. Les dimensions et la forme de l'ampoule interviennent aussi de la même manière.

On a coutume de rapporter une quantité d'émanation à la quantité de radium avec laquelle elle serait en équilibre. Il faut remarquer qu'une ampoule d'émanation qui, mesurée par les rayons $\gamma$, se montre égale en rayonnement à une ampoule de radium, contient non pas autant d'émanation que celle-ci, mais un peu moins, de sorte que l'on doit effectuer une petite correction négative, pour faire correspondre à l'émanation séparée qu'on mesure, une quantité de radium qui pourrait être en équilibrè avec elle. Cette correction, qui n'est que d'environ $0, \ddot{3} 0 / 0$, résulte du retard apporté par la transformation de l'émanation en dépôt actif. Le rayonnement $\gamma$ mesuré à un moment donné correspond non pas à la quantité d'émanation actuellement présente, mais à celle qui était présente quelques heures auparavant.

Enfin, dans certains cas, on utilise comme source de rayons le radium C que l'on a fait déposer sur un support convenable. La quantité de cette substance peut aussi être mesurée par le rayonnement $\gamma$ et évaluée par la quantité de radium avec laquelle elle pourrait être en équilibre.

Toutes ces mesures, qui peuvent être effectuées par l'emploi de rayons $\gamma$, sont rendues absolues quand on dispose pour les compa- 
raisons d'une ampoule étalon contenant une quantité connue de radium. La préparation d'une telle ampoule suppose que l'on a pu peser avec précision une petite quantité d'un sel de radium bien défini.

$$
*^{\star} *
$$

Examinons maintenant quels sont les travaux dans lesquels la quantité de radium utilisée joue un rôle important. Nous aurons d'abord à citer la détermination de diverses constantes qui doivent être rapportées à une quantité connue de radium :

Détermination de la quantité de chaleur émise par unité de temps par le radium et ses dérivés en équilibre avec lui. - Détermination de la quantité d'électricité positive émise sous forme de rayons $\alpha$. - Détermination de la quantité d'électricité négative émise sous forme de rayons $\beta$. - Détermination du nombre d'ions qui peuvent ètre produits au total par le rayonnement $\alpha$, le rayonnement $\beta$ et le rayonnement $\gamma$, ensemble et séparément. - Détermination du nombre de particules (ou rayons) a émises par le radium ou l'un de ses dérivés en équilibre avec lui, et de la charge de chaque particule. Évaluation de la fraction de l'énergie dégagée, mesurée sous forme de chaleur, qui est attribuable à l'énergie cinétique des rayons $\alpha$.

Détermination du rapport entre les proportions de radium et d'uranium dans les minéraux. Teneur en radium de la surface de la terre, dans les roches, les eaux des sources et de la mer. - Teneur de l'atmosphère en émanation du radium. - Évaluation de la quantité de chaleur dégagée par unité de temps dans le globe terrestre. Évaluation du nombre d'ions qui peut être produit dans l'atmosphère par les substances radioactives contenues dans le sol et l'atmosphère, et comparaison de ce nombre avec celui qui résulte de l'expérience directe.

Travaux d'ordre physiologique. Action du radium et de ses dérivés sur les tissus sains ou malades. L'effet dépend de la quantité de matière radioactive utilisée et du temps d'action.

Il y a de plus à considérer divers travaux pouvant être effectués avec des substances radioactives autres que le radium et ses dérivés, pour lesquelles on manque de base pour les mesures absolues, les substances n'ayant jamais été obtenues pures en quantité pondérable (radiothorium, actinium, etc.). On peut en ce cas avoir néanmoins 
LES MESLRES EN RADIOACTITTE ET LETALUN DU RADIUM 817

une base de comparaison, en rapportant la quantité de substance à une quantité de radium pouvant donner le mème effet ionisant dans des conditions déterminées. Ainsi le mésothorium peut ètre dosé par l'émission de rayons $\gamma$ par ses dérivés, mesurée dans des conditions déterminées et comparée à celle du radium.

Pour donner une base aux déterminations quantitatives dont on a eu un aperçu, divers laboratoires se sont servis d'étalons de radium obtenus en pesant soigneusement une petite quantité d'un sel supposé bien défini au point de vue chimique. En enfermant le sel dans une ampoule, on obtenait une ampoule étalon; en le dissolvant dans une quantité connue d'eau, on obtenait une solution étalon titrée.

Les sels utilisés étaient le chlorure ou le bromure.

La pureté d'un sel de radium ne peut être contrôlée que par la photographie du spectre et par la détermination du poids atomique du métal contenu dans le sel. Ces deux opérations entraînent toujours une petite perte de matière. la première pent être effectuée avec 1 milligramme de sel (méthode Demarçay); la deıxième demande quelques décigrammes et représente un travail délicat. Il ne suffit pas que le sel soit pur; il faut qu'il soit bien défini. Or les sels de radium éprouvent une transformation moléculaire sous l'influence de leur propre radiation et changent de composition. Cet effet est rapide avec le bromure. Le chlorure est plus stable; cependant, pour ètre assuré de la composition de ce sel, il faut lui faire subir des cristallisations avant la pesée.

On voit ainsi que la préparation d'étalons de radium ne peut être faite que dans les laboratoires qui possèdent assez de radium pour pouvoir entreprendre le travail nécessaire. Des étalons que l'on supposait approximativement exacts ont cependant été réalisés pour répondre aux besoins des mesures. Devant le nombre croissant des résultats expérimentaux numériques. obtenus avec une précision de mesures très satisfaisante, il devenait urgent de rendre comparables les résultats obtenus dans différents laboratoires afin de réaliser dans l'ensemble plus d'unité et de cohérence. Cette question a été soumise au Congrès de Radiologie, qui a été réuni à Bruxelles en septembre 1910.

Le Congrès a nommé une commission cumposée de savants qui, dans différents pays, travaillent dans le domaine de la radioactivité 
( $\mathrm{M}^{\mathrm{me}}$ Curie et A. Debierne pour la France, Rutherford et Soddy pour l'Angleterre, Hahn et Geitel pour l'Allemagne. St. Meyer et E. von Schweidler pour l'Autriche, Boltwood et Eve pour les États-Unis). La Commission a reconnu la nécessité de la préparation d'un étalon international de radium, consistant en une ampoule contenant environ 20 milligrammes de radium métal. L'exécution de cet étalon a été confiée à $\mathrm{M}^{\mathrm{me}}$ Curie, qui a accepté d'entreprendre ce travail. Il a. été décidé que, quand l'étalon international serait établi, des étalons secondaires seraient préparés pour les différents pays, et que lar teneur de ces étalons en radium serait fixée au moyen d'une comparaison par rayons y avec l'étalon international. Les solutions titrées devront être préparées avec des sels dont la teneur en radium aura été contrôlée par rapport à un étalon secondaire. Ces propositions de la Commission ont été adoptées par le Congrès. Celui-ci a encore pris quelques autres décisions :

L'unité dont l'usage a été adoptée est le gramme de radium métal, les propriétés radioactives du radium étant les mêmes à tous ses états moléculaires. L'unité du gramme étant pratiquement grande, on a envisagé l'utilité éventuelle d'une unité beaucoup plus petite, telle que $10^{-10}$ gramme de radium, à laquelle on donnerait un nom spécial.

L'émanation étant évaluée en fonction de la quantité de radium avec laquelle elle pourrait être en équilibre, il a paru utile d'établir sur cette base une unité d'émanation. L'unité adoptée est la quantité d'émanation qui est en equilibre radioactif avec 1 gramme de radium. Le Congrès a voulu donner à cette unité le nom de curie afin d'honorer la mémoire de Pierre Curie et son œuvre en radioactivité. La nouvelle unité est déjà entrée en usage; sa millième partie, un millicurie, est une unité pratique. d'un ordre de grandeur convenable dans les laboratoires. Mais une unité beaucoup plus. petite, $10^{-10}$ curie par exemple, pourrait recevoir un nom spécial pour être utilisée, comme l'unité correspondante de radium, dans les travaux sur la radioactivité du sol et des eaux.

On pourrait évidemment concevoir que la quantité d'émanation fût donnée par son poids, mais ce genre d'indication serait moins. approprié à l'usage. De plus il ne saurait en être question actuellement, la pesée d'une quantité d'émanation qui est en équilibre avec une quantité donnée de radium étant une opération très difficile qui n’a pas encore pu être effectuée avec une grande précision. 
Le Congrès de Bruxelles a chargé la Commission de prendre les dispositions nécessaires pour fixer l'usage de l'étalon international.

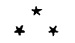

L'étalon international a été préparé au courant du mois d'août 1911. Le sel utilisé était pris sur la provision de chlorure de radium très pur d'environ $0^{\text {gr }}, 4$ qui avait été préparé par moi en 1907 en vue de la détermination du poids atomique du radium. La très grande pureté de ce sel avait été mise en évidence par l'aspect du spectre; le poids atomique obtenu est $226, \%$. Le sel avait été conservé soigneusement à l'abri de tout accident; une partie avait été utilisée pour la préparation du radium métallique et de solutions étalons.

Voici quelles ontété les opérations effectuées pour amener le sel à un état défini. Le sel a été additionné d'eau contenant un peu d'acide chlorhydrique; il s'y est dissous presque complètement, laissant un très faible résidu de sel insoluble, tel qu'il s'en forme toujours à la longue, probablement par suite de l'attaque des vases, même dans une enceinte desséchée. Le résidu ayant été séparé par filtration, on a ajouté à la solution limpide une très petite quantité d'acétate de plomb, puis on a traité la solution par l'hydrogène sulfuré. Le sulfure de plomb, en précipitant, devait entraîner la totalité du plomb radioactif (radium D), de radium $\mathrm{E}$ et de polonium (radium F) formés dans le sel de radium pendant 4 ans. Les sulfures ayant été séparés par filtration, la solution a été évaporée à sec. Le sel a été redissous dans un mélange d'eau et d'acide chlorhydrique pur et la solution a été concentrée de manière à cristalliser. Après refroidissement, l'eau mère a été décantée et les cristaux ont été lavés à l'acide chlorhydrique pur concentré, - puis encore redissous dans un mélange d'eau et d'acide pur afin d'obtenir une nouvelle cristallisation. Celle-ci était très belle et a donné quelques gros cristaux très allongés en forme d'aiguille. L'eau mère assez abondante, la cristallisation ayant eu lieu en milieu très acide, a été décantée. Les cristaux ont été séchés au bain-marie d'abord, à l'étuve réglée à $150^{\circ}$ ensuite. Cette température est suffisante pour ramener le sel à l'état anhydre et à la formule $\mathrm{RaCl}^{2}$.

On a procédé à la préparation de l'ampoule. Celle-ci a reçu la forme d'un tube de verre très étroit à paroi mince: diamètre intérieur 
$0^{\mathrm{mm}}, 9$; épaisseur du verre, $0^{\mathrm{mm}}, 2 \bar{\tau}$; verre blanc. Le fond du tube a été étiré et fermé à la lampe sur un fil très mince en platine pénétrant à l'intérieur. Ce fil a pour but d'empêcher l'accumulation de charges électriques à l'intérieur de l'ampoule, en établissant une communication conductrice à l'extérieur. Un petit bouchon de verre s'adaptant sur le tube a été préparé, et l'ampoule avec son bouchon a été soigneusement pesée. 11 était à craindre que le sel n’absorbe de la vapeur d'eau pendant l'opération de remplissage et que l'eau ne puisse ensuite être chassée facilement, eu égard à la forme del'ampoule. Pour éviter ce danger, on a effectué le remplissage dans une atmosphère chaude. La capsule contenant les cristanx écrasés avec un agitateur a été placée sur une plaque métallique épaisse et chauffée. La température de l'air avoisinant le sel était d environ $80^{\circ}$. L'ampoule à remplir séjournait constamment dans cet air chaud. A près avoir été remplie suffisamment, elle a été recouverte du bouchon et soumise à des pesées alternant avec des séjours à l'étuve à $150^{\circ}$, suivis de retour à la température ordinaire dans un dessiccateur. Dans ces opérations, le poids de l'ampoule et de son contenu restait constant à un cinquantième de milligramme près. L’ampoule a alors été fermée à la lampe à une petite distance au-dessus du niveau du sel qui occupe la longueur totale de 32 millimètres. On s'est assuré qu'il ne restait pas de sel après la partie détachée, en quantité appréciable à la balance. Le remplissage a pu être effectué dans d'aussi bonnes conditions, sans adhérence de sel aux parois, grâce au dessèchement parlait de la matière. L’ampoule est représentée dans la fig. 4 .

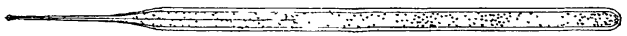

Fig. 4 .

La balance utilisée était une balance Curie à amortissement rapide et à microscope, réglée de manière à être sensible au centième de milligramme. Les poids utilisés étaient des poids de précision étalonnés. Une ampoule semblable à l'ampoule principale servait de tare.

Le sel avait atteint un état défini et un poids constant égal à $0^{\mathrm{gr}}, 02199$ de chlorure anhydre $\mathrm{RaCl}^{2}$, à la précision de un cinquantième de milligramme environ. Le poids de l'ampoule est $0^{\text {gr }}, \mathbf{1 0 2 2}$. La quantité de radium métal contenu dans l'ampoule est $0^{\mathrm{gr}}, 0167 \mathrm{3}$. 
LES MESURES EN RADIOACTIVTÉ ET LETALON DL RADILM 821

Grâce à la minceur du verre et à la forme allongée de l'ampoule, la proportion de rayons $\gamma$ absorbée dans le sel et dans l'enveloppe du verre ne doit pas atteindre $0,30,0$.

Le sel occupe presque intégralement la longueur de l'ampoule, et c’est là une disposition utile pour la précision des mesures qui peuvent être quelque peu influencées par les changements de position du sel, lors des trépidations. En revanche, on peut se demander si en restreignant ainsi l'espace libre l'on ne risque pas de compromettre la sécurité de l'ampoule qui pourrait éclater par la pression des gaz qui s'y accumulent progressivement. Mais le sel ayant été très soigneusement privé de toute son eau de cristallisation, la production de gaz tonnant, phénomène toujours observé avec le sel hydraté, n'est pas à craindre. On a donc seulement à envisager la production d'hélium, qui ne peut ètre évitée et qui a lieu en raison d'environ $\mathcal{Q}^{\text {n } \mathrm{m}^{3},}, \breve{3}$ par année pour la quantité de radium présente dans l'ampoule. Le voJume de celle-ci étant environ $20 \mathrm{~mm}^{3}$ et le volume réellement occupé par la matière des grains ne pouvant guère dépasser $6 \mathrm{~mm}^{3}$, un temps de huit années est nécessaire pour que la pression de l'hélium formé égale la pression atmosphérique, le gaz étant supposé libre. (En réalité, le gaz reste occlus dans le sel, mais il peut être dégagélors d'un échauffement accidentel de l'ampoule.) L'ampoule ayant été fermée à chaud, la pression de l'air au début, après refroidissement, était d'ailleurs inférieure à la pression atmosphérique. On peut prévoir que dans quinze ans il pourra être utile d'ouvrir l'extrémité effilée de l'ampoule afin de laisser échapper le gaz hélium en excès.

En même temps que l'étalon international était préparé en France, un travail analogue était effectué à l'Institut du Radium de Vienne où quelques ampoules étalons, contenant des quantités différentes de chlorure de radium anlıydre ont été faites avec un sel soigneusement purifié appartenant à l'Académie des Sciences de Vienne.

Il a paru alors possible de prendre des décisions définititives au sujet des étalons et de réunir, à cet effet, la Commission qui avait été chargée de ce travail par le Congrès de Bruxelles.

La réunion de la Commission internationale a eu lieu à Paris à la 
fin de mars 1912. Sept membres étaient présents. Les occupations de la Commission ont consisté à comparer entre eux les divers étalons préparés et à fixer les règles de leur emploi.

La comparaison des étalons a été faite par la mesure du rayonnement $\gamma$, et l'installation des appareils de mesures a été assurée par le Laboratoire de Radioactivité de la Faculté des Sciences de Paris, sous la direction de M. Debierne. Cependant l'installation n'a pas été faite dans ce Laboratoire, car dans les salles de celui-ci l'air possède une conductibilité spontanée bien plus élevée qu'à l'état normal, et cette circonstance est défavorable aux mesures précises. Grâce à l'obligeance de M. le Professeur Lippmann, les appareils ont pu être installés dans une salle inactive appartenant à son service.

La comparaison a été faite par deux méthodes différentes. La première est une méthode de compensation employée au Laboratoire de Paris; elle consiste à utiliser un grand condensateur à plateuux (fo. 3, III) spécialement construit pour cette circonstance. Voici quelles étaient les dimensions de cet appareil :

Dianètre du plateau E............... $50 \mathrm{~cm}$.

Diamètre de la boite cylindrique $\mathrm{P} \ldots \ldots \ldots \ldots .52 \mathrm{~cm}$.

Distances du plateau E aux bases de la boîte... $\quad 3,5$ et $2 \mathrm{~cm}$.

Un écran de plomb de 1 centimètre d'épaisseur était posé sur la base supérieure de la boîte $\mathrm{P}$. L'ampoule à examiner était placée sur une feuille de papier posée sur l'écran. Le courant produit dans la chambre était mesuré au moyen d'un dispositif composé d'un électromètre et d'un quartz piézoélectrique. On étudiait soigneusement l'influence des déplacements de l'ampoule sur la valeur du courant. Cette influence est inappréciable pour de petits déplacements à partir de la position centrale.

La deuxième méthode, dont le montage a été fait conformément aux indications de M. Rutherford, est une méthode de compensation d'un genre différent. Un courant constant produit dans une chambre d'ionisation auxiliaire contenant de l'uranium est compensé par le courant obtenu dans la chambre d'ionisation principale (type $f g .3, \mathrm{I}$ ) par l'action de l'ampoule placée à une distance convenable. L'éloignement de l'ampoule dépendant de sa teneur en radium, il est nécessaire de connaître la loi de cette dépendance pour comparer entre elles des quantités de radium différentes. Avec cette méthode, les con- 
LES MESURES EN RADIOACTIVITÉ ET L'ÉTALON DL RADILM 823 ditions ne sont pas aussi exactement pareilles pour les ampoules à comparer qu'avec la précédente.

Les deux méthodes ont donné des résultats très concordants et l'on a trouvé de plus entre les différents étalons une conco rdance extrêmement satisfaisante, les écarts étant de l'ordre des erreurs expérimentales, estimées à $0,20 / 0$ environ.

Ce résultat obtenu avec des étalons préparés d’une part en France, d'autre part en Autriche, en partant de sels purs préparés aussi d'une manière indépendante dans ces deux pays, à partir d'un même minerai, est tout à fait remarquable. Il prouve à la fois la parfaite sécurité qu'on peut avoir relativement à la composition du chlorure de radium anhỹdre et aussi la grande perfection des méthodes de comparaison basées sur la mesure des rayons $\gamma$. Ainsi la base pour les mesures des teneurs en radium par ce moyen se trouve établie de maniière à inspirer toute confiance.

La comparaison a été facilitée par cette circonstance que l'épaisseur du verre des ampoules est non seulement faible, mais encore la même pour les différents étalons.

A la suite de ce travail la Commission a pris les décisions suivantes :

$1^{\circ}$ L'étalon préparé par $\mathrm{M}^{\text {me }}$ Curie a été choisi comme étalon international, et il a été décidé que cet étalon serait déposé à Paris, au Bureau international des Poids et Mesures, avec le consentement du Directeur de cette institution.

L'étalon international ne devra être utilisé que pour la mesure d'étalon s secondaires destinés aux différents pays.

$2^{\circ}$ Un des étalons préparés à Vienne sera conservé dans l'Institut de Vienne, à titre d'étalon auxiliaire $\left(31^{\mathrm{mg}}, 17 \mathrm{RaCl}^{2}\right)$.

$3^{\circ}$ Les étalons secondaires seront préparés avec 10 à 40 milligrammes de sel contenant au moins $900 / 0$ de radium et auront une forme semblable à celle des étalons de Vienne (1). Ils seront soumis à un e mesure d'abord à Vienne, ensuite à Paris, et recevront un certificat indiquant leur teneur en radium.

$4^{\circ}$ Le radium utilisé pour l'étalon international appartenant à $\mathbf{M}^{\mathrm{me}}$ Curie, la Commission décide de faire des démarches pour qu'une

(1) Les ampoules de Vienne ont $3^{\mathrm{mm}}, 2$ de diamètres et le sel n'occupe qu'une petite partie du volume. La forme de l'étalon international est plus parfaite, mais plus difficile à réaliser. 
quantité équivalente de radium soit remise à $M^{\text {me }}$ Curie en échange par les pays intéressés 1 .

Il nous reste à examiner l'état actuel de l'étalon international, discuter les lois de l'évolution prévue et leur influence sur l'usage de l'étalon.

Au point de vue chimique, le sel qui constitue l'étalon était, an moment de la fermeture de l'ampoule, un chlorure de radium pur et anhydre répondant à la formule $\mathrm{RaCl}^{2}$. Au point de vue radioactif, le sel est devenu, dès sa cristallisation. le siège d'une accumulation de l'émanation et des dérivés successifs. A ce point de vue l'état du sel est devenu approximativement stationnaire en un mois; les quantités d'émanation, de radium $\mathrm{A}, \mathrm{B}, \mathrm{C}$, qui se trouvent maintenant dans l'ampoule, en équilibre radioactif avec le sel, sont très faibles (au-dessous d'un dix-millième de milligramme); néanmoins la part de ces matières dans l'émission du rayonnement est considérable; le rayonnement $\gamma$ en particulier est attribuable au radium C. Au ccurs de l'évolution lente qui a continué ensuite, s'accumulent les substances $\mathrm{D}, \mathrm{E}$ et $\mathrm{F}$ dont la proportion augmente pendant un temps de l'ordre de cent ans. Parmi ces substances, seul le radium $E$ semble donner naissance à une émission de rayons $\gamma$; mais ces rayons sont beaucoup moins pénétrants que ceux du radium $C$, et l'on peut penser qu'ils n'interviennent guère dans les mesures dans lesquelles on utilise les rayons qui ont traversé une épaisseur de plomb suffisante ( 1 centimètre); ce point aurait d'ailleurs besoin d'une vérification rigoureuse. Si l'on admet provisoirement qu'il n'y a pas à tenir compte des produits à évolution lente, nous devons nous attendre à voir les rayons $\gamma$ très pénétrants suivre la loi de l'évolution du radium, c'est-à-dire décroître suivant une loi exponentielle avec une période de 2000 ans environ. D'après cela la décroissance en un an serait denviron $0,040 / 0$, de sorte que pendant dix années l'étalon pourra être considéré comme invariable et que pendant vingt ou trente ans encore ensuite, une petite correction approximativement connue permettrait de continuer son emploi ; c'est suffisant pour qu'il rende les services qu'on en espère.

On peut se demander maintenant si le radium provenant de mine-

(1) Aussitôt que le texte des délibérations a été publié, cette question a été résolue. Le $\mathrm{D}^{\mathrm{r}}$ et $\mathrm{M}^{\mathrm{ne}}$ G. P. Beilby ont offert à la Commission d'assumer les frais de remplacement de l'étalon international et ont bien voulu donner à leur proposition le caractère d'un témoıgnagre particulier d'estime à mon égard dont je les remercie bien sincirement. 
LES MESLRES EN RADIOACTIVTTÉ ET L'ÉTALON DL RADILM 82:

rais d'urane ne pourrait contenir en quantité impondérable une trace de substance radioactive autre que ses dérivés et capable d'émettre des rayons $\gamma$ très pénétrants. Parmi de telles substances le mésothorium est surtout à craindre. Ce corps est un dérivé dı thorium, lequel est, en général, présent dans les minerais d urane. Le mésothorium accompagne le radium dans les séparations chimiques effectuées sur le minerai et dans la cristallisation fractionnée. Cette substance donne naissance à une série de dérivés, suivant une évolution longue et complexe, comportant un terme final qui donne lieu à une émission de rayons $\gamma$ comparables en pouvoir pénétrant à ceux du radium C. La présence de mésothorium dans le radium est donc de nature à altérer la loi d'évolution du rayonnement $\gamma$ et à fausser le dosage du radium par ce rayonnement. Fort heureusement il existe des minerais d'urane dont la teneur en thorium est très faible et qui, pour cette raison, ne peuvent contenir du mésothorium qu'en proportion trop petite pour donner lieu aux difficultés ci-dessus. Telle est la pechblende de Saint-Joachimsthal, qui contient environ 1 kilogramme de thorium dans 50000 kilogrammes de minerais à $33 \%$ d'oxyde d'urane, pouvant fournir $3^{\mathrm{gr}}, \mathbf{7}$ de radium $\left({ }^{1}\right)$. Le rayonnement $\gamma$ de 1 kilogramme de thorium étant absolument négligeable par rapport à celui de $3^{\mathrm{g} r}, 7$ de radium (de l'ordre de 3 cent-millièmes), la présence du mésothorium ne peut en ce cas constituer un inconvénient appréciable. C'est précisément le cas de l'étalon international et des étalons de Vienne, lesquels ont été tous préparés avec des sels de radium extraits de la pechblende de Saint-Joachimsthal.

On voit ainsi que l'emploi de l'étalon international peut avoir lieu dans des conditions de grande sécurité. On peut de plus augmenter cette sécurité en préparant dans des conditions analogues une ampoule dont l'activité sera suivie en fonction du temps au moyen d'un appareil de mesures absolues (par exemple, par la méthode du quartz piézoélectrque). On pourra ainsi connaître, par l'expérience, la loi d'évolution de l'étalon avec le temps.

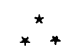

En résumé, l'adoption d'un étalon exactement connu doit rendre les services suivants:

(1) St. Meyer et Hess, Acad. des Sciences de Vienne, 1912. 
Au point de vue de la science de la radioactivité :

Assurer l'accord entre les résultats numériques obtenus dans différents laboratoires et faisant intervenir une quantité de radium donnée. En cas de désaccord, provoquer la revision des travaux considérés, ce qui peut conduire à l'observation de faits nouveaux.

Au point de vue des applications médicales:

Assurer un emploi judicieux des propriétés physiologiques du rayonnement, en permettant de doser exactement et en toute sécurité l'intensité de la source de rayons utilisée.

Au point de vue industriel :

Donner de la stabilité et de la sécurité à l'industrie du radium en permettant de vendre des produits à teneur en radium exactement connue, de manière à établir une confiance entière entre les industriels et les acheteurs. Ce service peut être étendu à la vente de mésothorium ainsi que je l'ai indiqué plus haut.

On peut se rendre compte par l'ensemble des considérations développées dans cet article que l'œuvre de l'Etalon international de Radium a un caractère de haute utilité générale. On est en droit d'espérer que l'initiative prise à ce sujet par le Congrès de Radioactivité de 1910 sera féconde en conséquences heureuses représentant un progrès réel dans des directions variées. 